\title{
CHARACTERIZATION OF SUMOYLATED PROTEINS IN HUMAN SPERM
}

Sara Marchiani ${ }^{1}$, Beatrice Ricci ${ }^{1}$, Lara Tamburrino ${ }^{1}$, Monica Muratori ${ }^{1}$, Marta Cambi ${ }^{1}$, Daniele Nosi ${ }^{2}$, Gianni Forti ${ }^{1}$, Elisabetta Bald ${ }^{1}$

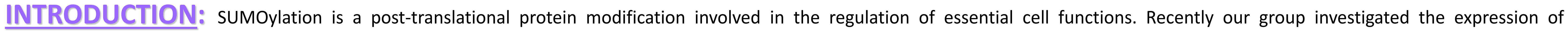

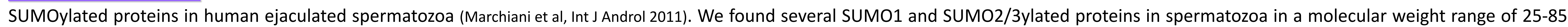

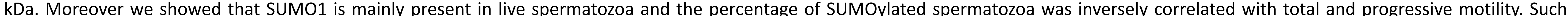

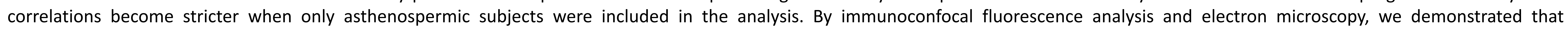
SUMOylated proteins are mainly located in the nucleus and in the midpiece.

AIM OF THE STUDY (1): To characterize possible SUMO1 target proteins in human spermatozoa. In particular we evaluated whether SUMOylation occurs in the three proteins:

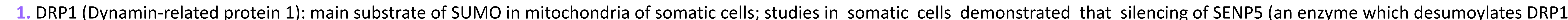
leads to stabilization of DRP1, to a fragmented mitochondrial morphology and to an increased production of ROS (Harder et al, Curr Biol. 2005; Zunino et al, J Cell Science. 2007)

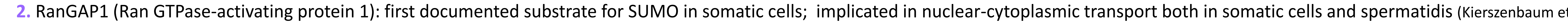
al, Mol Reprod Dev. 2002)

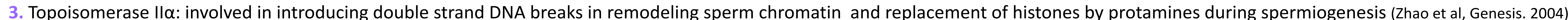
oxidative and heat stress increased SUMO-Topoisomerase II levels in germ cells (Shrivastava et al, Reproduction. 2010)

\section{RESULTS \& CONCLUSIONS (AIM 1)}

\section{DRP1: Immunoprecipitation and western blot experiments}

DRP1 in TL (total sperm lysate) and SUMO1 immunoprecipitated (IP) sperm proteins in normo- and astheno-spermic pools

IB: DRP

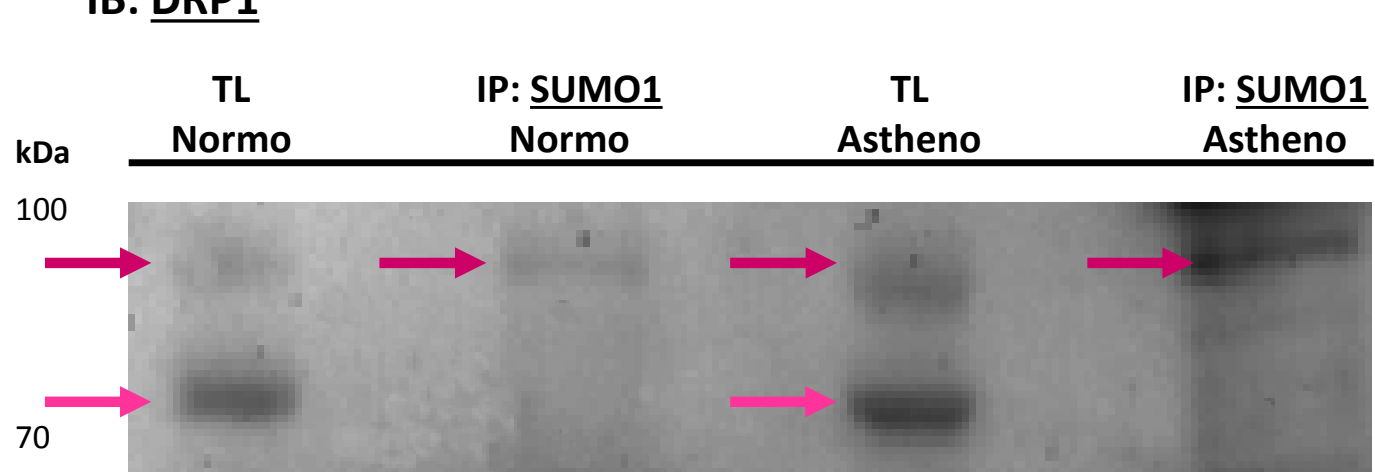

$\underset{\text { DRP1 } 1}{\mathrm{SUMM} 1 \mathrm{ylted} \text { DRP1 }}$

\section{$>$ DRP1: Confocal microscopy experiments}

Normozoospermic subject
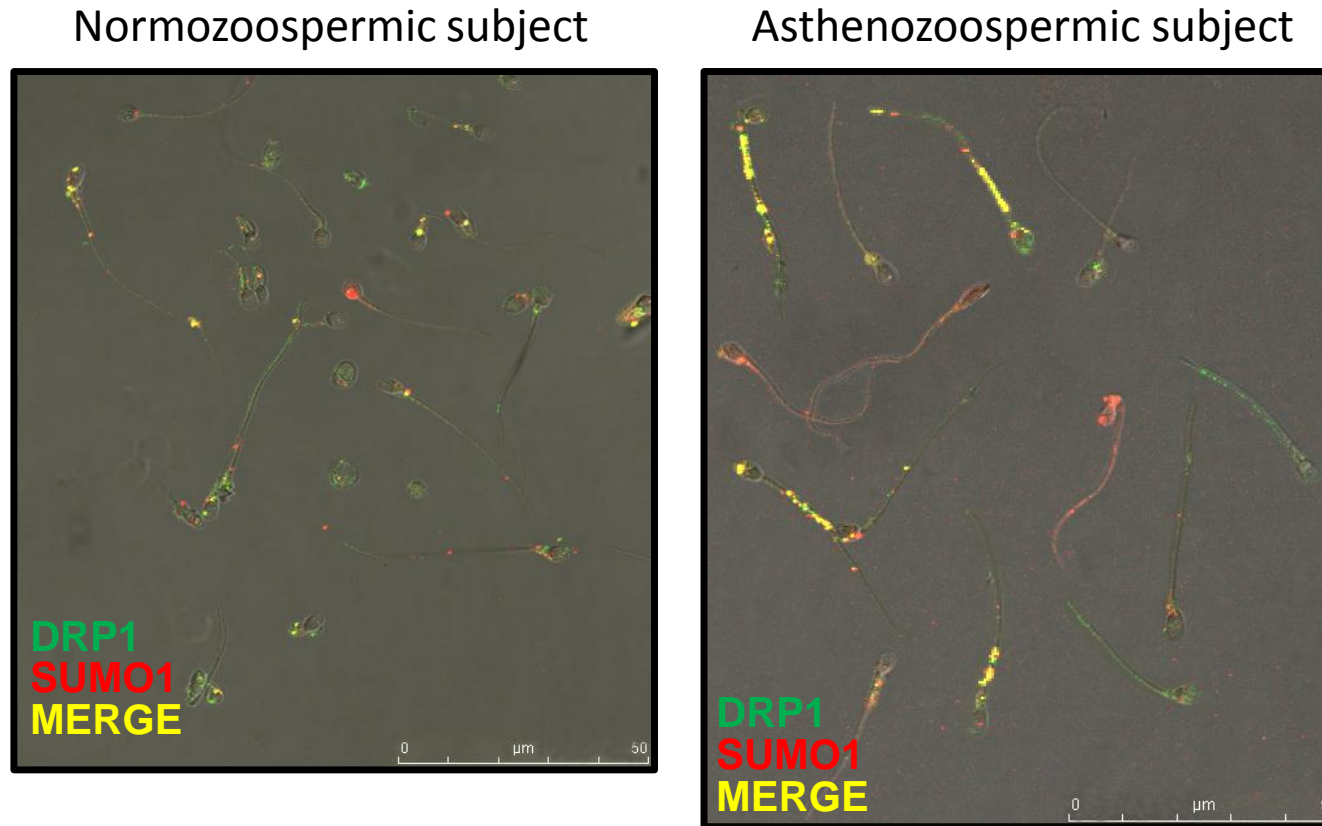

Sperm showing DRP1-SUMO1 midpiece colocalization /Total DRP1 positive sperm (\%): $50,03 \pm 6,32(n=5)$ vs $30,41 \pm 20,09(n=5), p=0,07$

Sperm with normal morphology/Total SUMO1 positive sperm (\%) vs $20,74 \pm 9,99$ vs $80,07 \pm 9,6(n=10), p<0,001$

DRP1 results to be sumoylated in human sperm

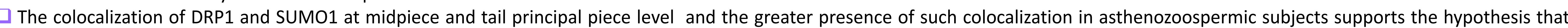
SUMOylation of DRP1 could impair mitochondrial function and consequently decrease the sperm motility

$\square$ Sperm with morphological defects seem to express higher SUMO fluorescence in agreement with a previous study on human spermatozoa (Vigodner et al, Hum Reprod. 2012)

\section{RanGAP1: Immunoprecipitation and western blot experiments}

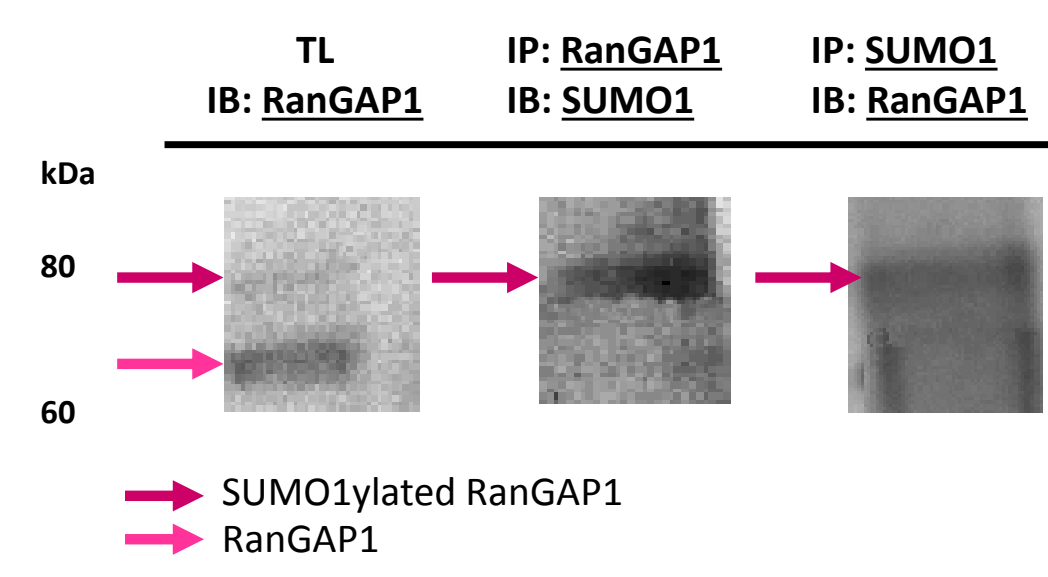

\section{RanGAP1: Confocal microscopy experiments}
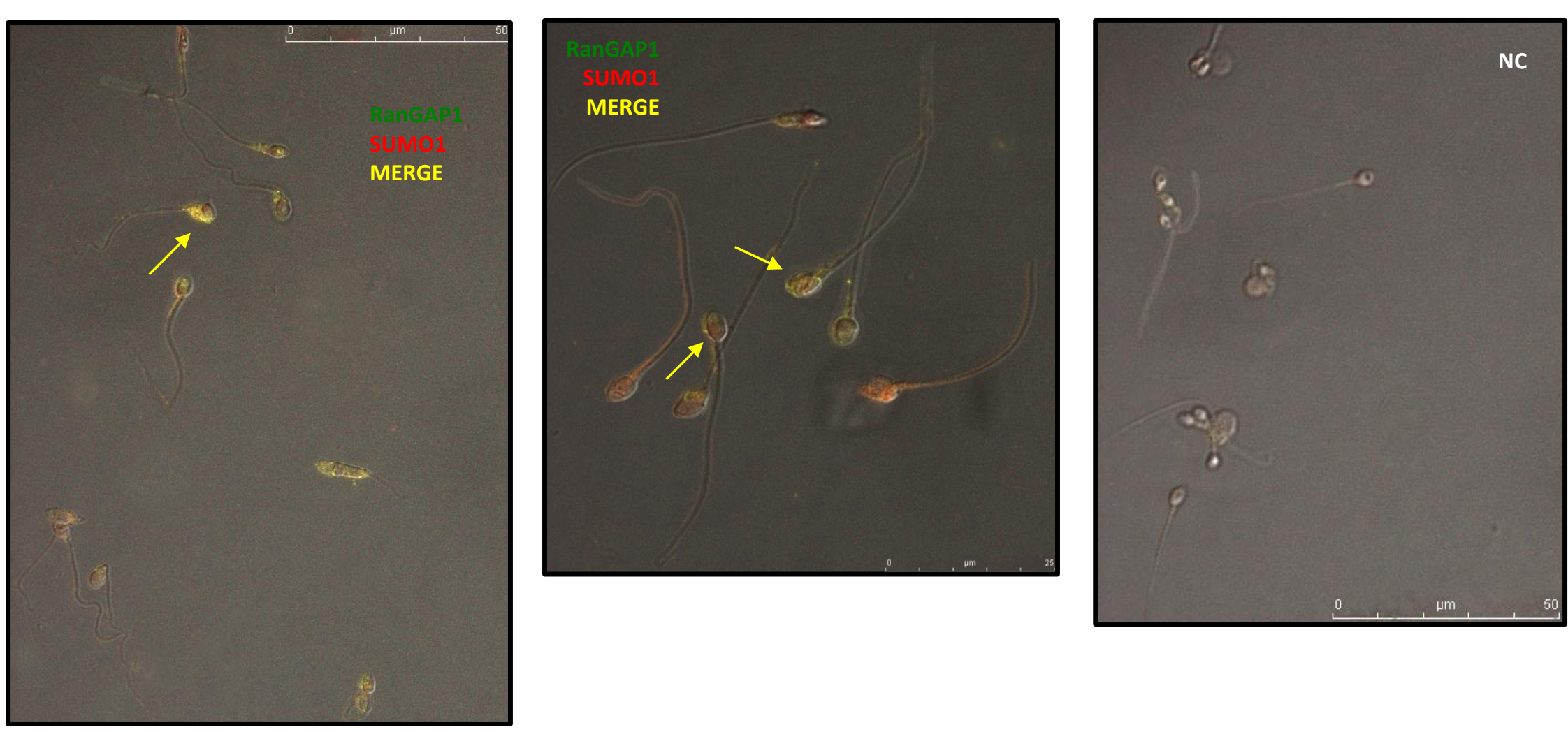

$\square$ RanGAP1 results to be sumoylated in human spermatozoa

$\square$ RanGAP1 and SUMO1 mainly localized at nucleus level

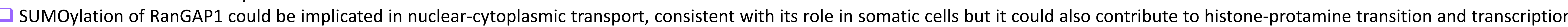
repression, as suggested by Vigodner group ( Vigodner et al, Developmental Biology. 2005)

\section{Topoisomerasella: Immunoprecipitation and western blot experiments}

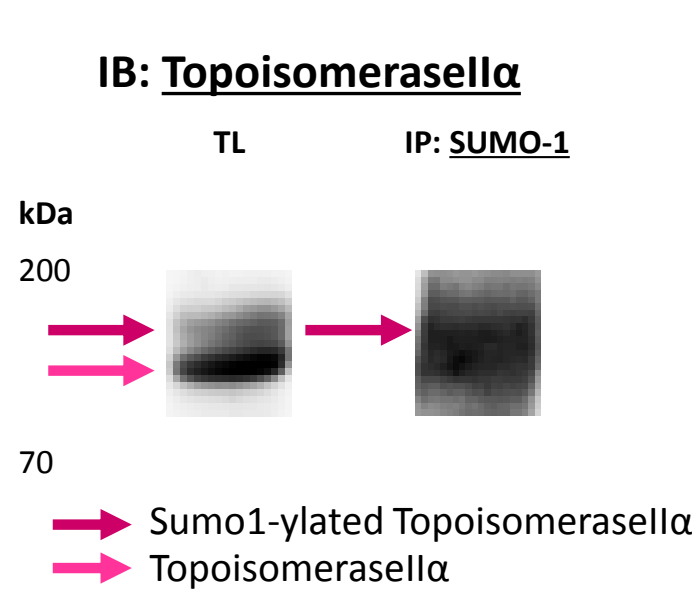

\section{Topoisomerasella: Confocal microscopy experiments}
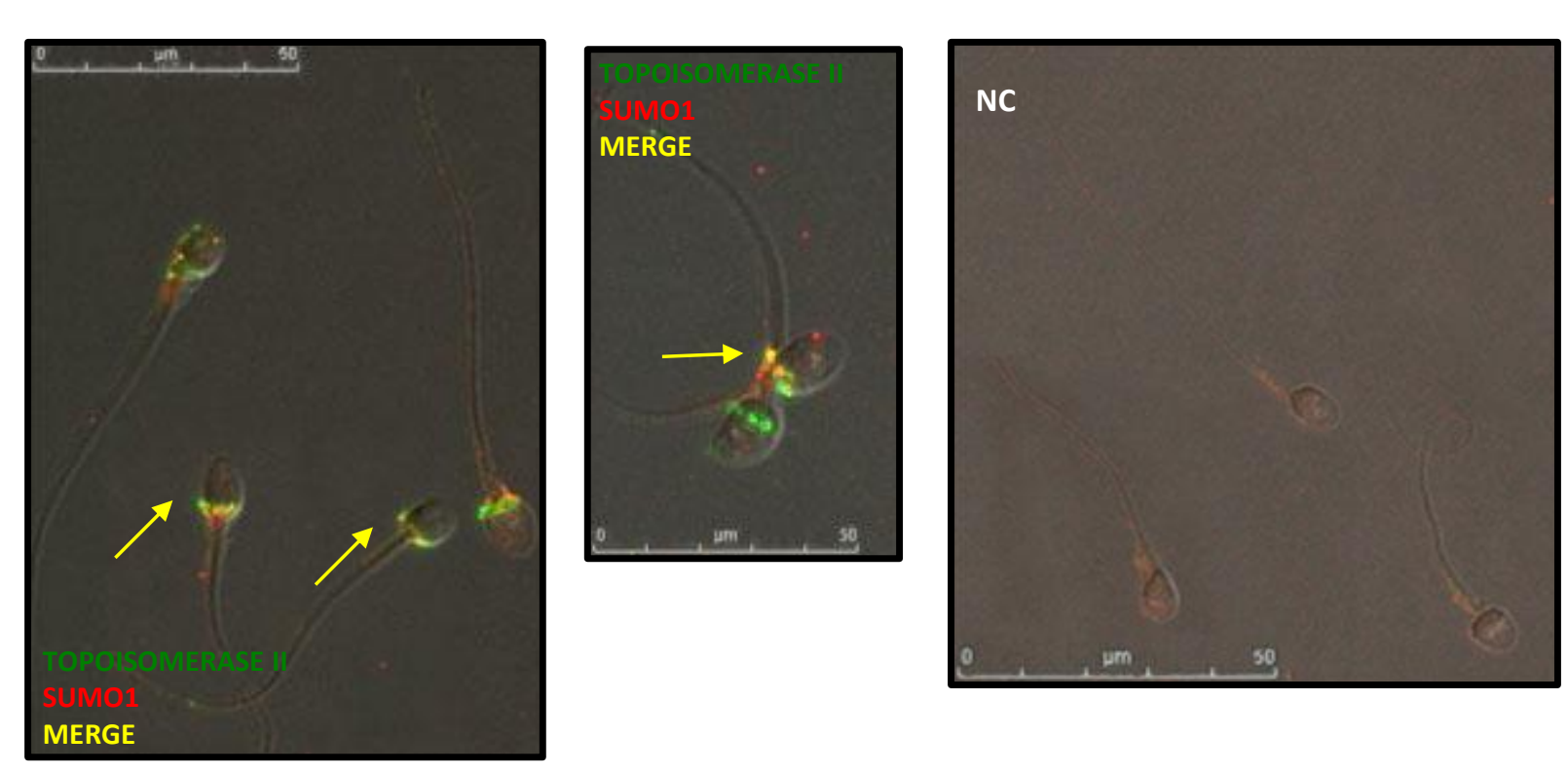

$\square$ Topoisomerasella is a SUMO substrate in mature spermatozo

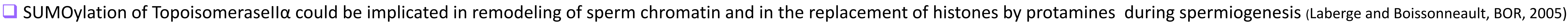

$\square$ Moreover the modification of this nuclear protein could play a role in the re-organization of sperm chromatin after fertilization (Bizzaro et al, Zygote, 2000)

AIM OF THE STUDY (2): To investigate the relationship between SUMO1ylation and sperm DNA fragmentation, since SUMO-modified proteins have been detected close to DNA strand breaks in nuclei of somatic cells and mouse spermatocytes

\section{RESULTS \& CONCLUSIONS (AIM 2):}

Signifcant positive correlation between SUMO1 and DNA fragmentation

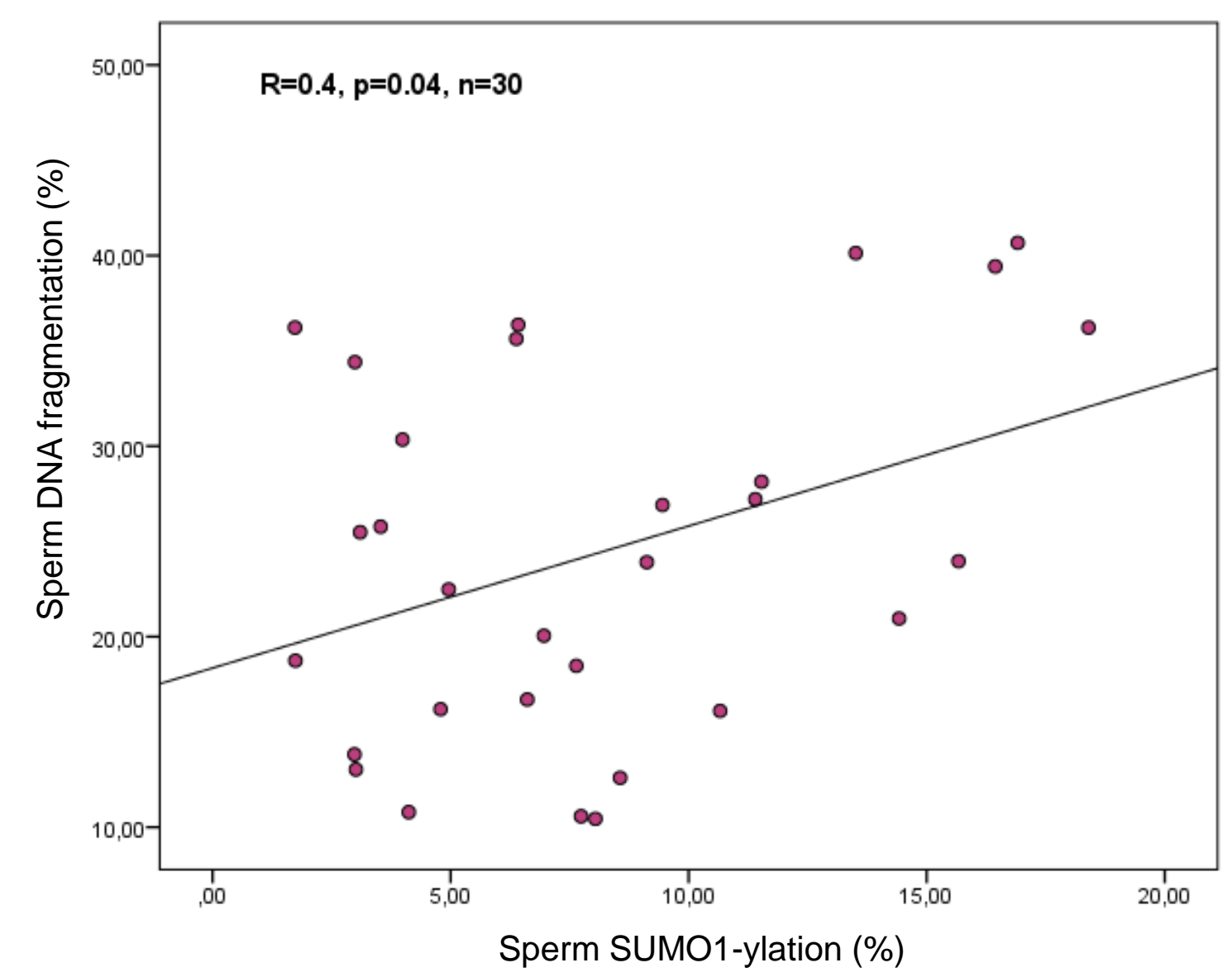

SUMO1 and DNA fragmentation are simultaneously found in live sperm

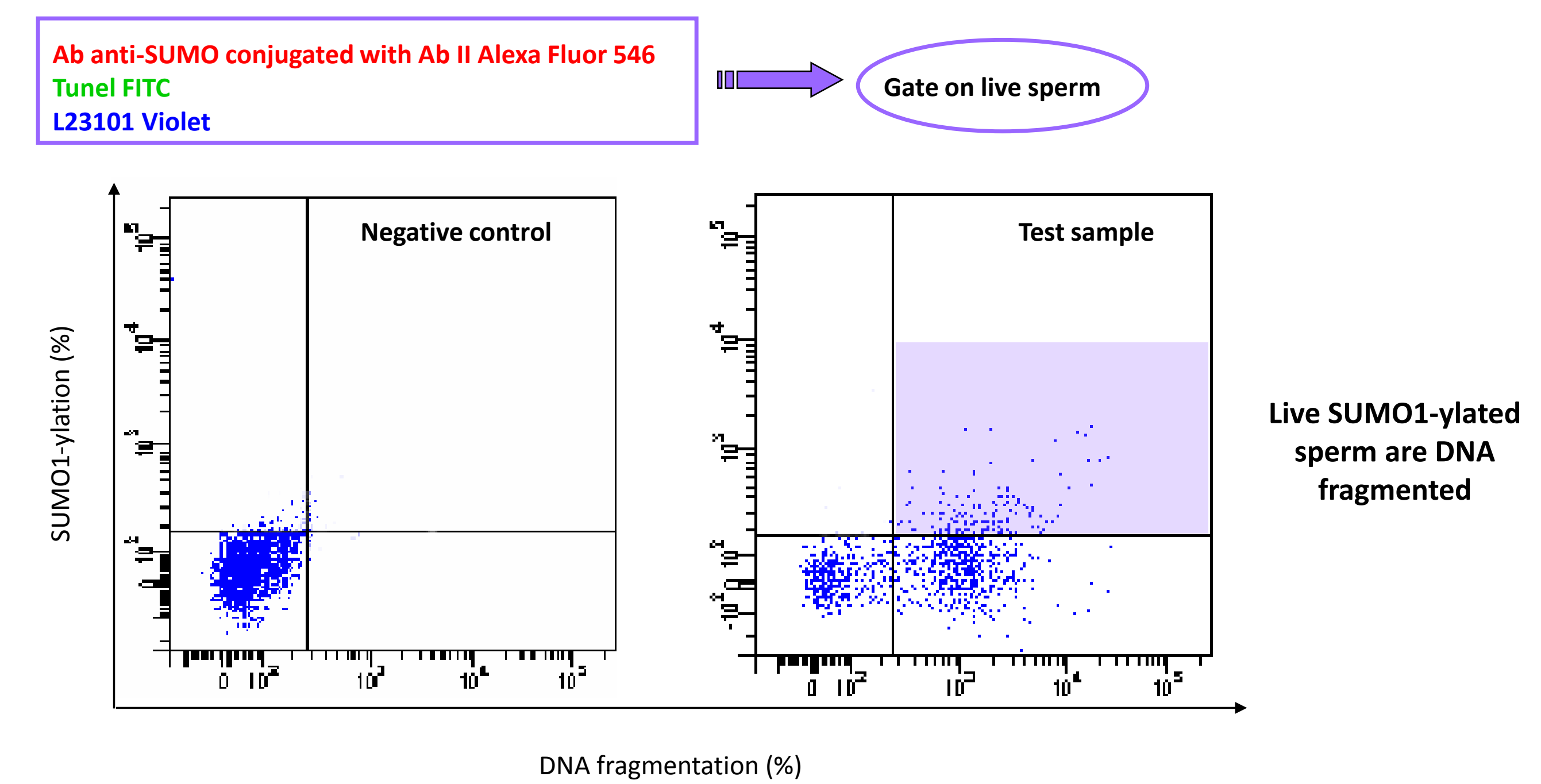

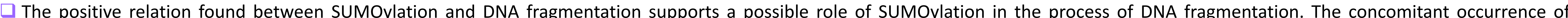

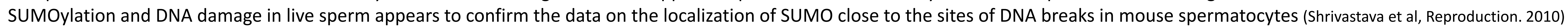
$\square$ Overall these data suggest a possible involvement of SUMOylated proteins in formation or repair of DNA breaks, with a mechanisms to be clarified 